\title{
BMJ Open Design of a randomised controlled trial of adapted physical activity during adjuvant treatment for localised breast cancer: the PASAPAS feasibility study
}

\author{
M Touillaud, ${ }^{1}$ A-M Foucaut, ${ }^{1,2}$ S E Berthouze, ${ }^{2}$ E Reynes, ${ }^{2}$ A-S Kempf-Lépine, ${ }^{1}$ \\ J Carretier, ${ }^{1,3}$ D Pérol, ${ }^{4} \mathrm{~S}$ Guillemaut, ${ }^{4} \mathrm{~S}$ Chabaud, ${ }^{4} \mathrm{~V}$ Bourne-Branchu, ${ }^{4}$ \\ L Perrier, ${ }^{1,5} \mathrm{O}$ Trédan, ${ }^{6}$ B Fervers, ${ }^{1,3} \mathrm{P}$ Bachmann ${ }^{7}$
}

To cite: Touillaud M, Foucaut A-M, Berthouze SE, et al. Design of a randomised controlled trial of adapted physical activity during adjuvant treatment for localised breast cancer: the PASAPAS feasibility study. BMJ Open 2013;3:e003855. doi:10.1136/bmjopen-2013003855

- Prepublication history for this paper is available online. To view these files please visit the journal online (http://dx.doi.org/10.1136/ bmjopen-2013-003855)

Received 20 August 2013 Accepted 25 September 2013

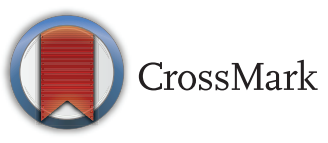

For numbered affiliations see end of article.

Correspondence to Dr Patrick Bachmann; patrick.bachmann@lyon. unicancer.fr

\section{ABSTRACT}

Introduction: After a diagnosis of localised breast cancer, overweight, obesity and weight gain are negatively associated with prognosis. In contrast, maintaining an optimal weight through a balanced diet combined with regular physical activity appears to be effective protective behaviour against comorbidity or mortality after a breast cancer diagnosis. The primary aim of the Programme pour une Alimentation Saine et une Activité Physique Adaptée pour les patientes atteintes d'un cancer du Sein (PASAPAS) randomised controlled trial is to evaluate the feasibility of implementing an intervention of adapted physical activity (APA) for 6 months concomitant with the prescription of a first line of adjuvant chemotherapy. Secondary aims include assessing the acceptability of the intervention, compliance to the programme, process implementation, patients' satisfaction, evolution of biological parameters and the medicoeconomic impact of the intervention.

Methods and analysis: The study population consists of 60 women eligible for adjuvant chemotherapy after a diagnosis of localised invasive breast cancer. They will be recruited during a 2-year inclusion period and randomly allocated between an APA intervention arm and a control arm following a 2:1 ratio. All participants should benefit from personalised dietetic counselling and patients allocated to the intervention arm will be offered an APA programme of two to three weekly sessions of Nordic walking and aerobic fitness. During the 6-month intervention and 6-month follow-up, four assessments will be performed including blood draw, anthropometrics and body composition measurements, and questionnaires about physical activity level, diet, lifestyle factors, psychological criteria, satisfaction with the intervention and medical data.

Ethics and dissemination: The study was approved by the French Ethics Committee (Comité de Protection des Personnes Sud-Est IV) and the national agencies for biomedical studies and for privacy. All participants will give written informed consent. The study findings will be disseminated through the scientific public and serve as a foundation for future randomised controlled trials of efficacy.

\section{ARTICLE SUMMARY}

Strengths and limitations of this study

- This study will provide feasibility of implementing an innovative 6-month programme of adapted physical activity during breast cancer treatment for a future full-scale randomised controlled trial testing the effectiveness of physical activity on survival-related outcomes.

- The physical activity programme presented has the advantage of being performed during breast cancer adjuvant treatment, personalised and supervised by trained professionals. It reflects growing evidence showing the necessity to maintain or start physical activity as soon as possible after a cancer diagnosis and avoid loss of physical fitness.

- As in most feasibility studies, the sample size is small but it is sufficient to test procedure adequacy for formalising the design of a definitive trial.

\section{INTRODUCTION}

Throughout the world, breast cancer is the leading cause of cancer in women and the leading cause of cancer death, with c. 1383500 new cases and 458400 deaths in 2008. ${ }^{1}$ Thanks to progress in diagnostic tools, care and treatments in Western countries, survival after diagnosis is greater than $80 \%$ at 5 years. $^{2}$ Overall, breast cancer seems to be a disease of the developed world and particularly affects Western countries or those with a Western lifestyle. Therefore, it is important for patients with breast cancer and survivors to maintain optimal health and minimise the deleterious effects of cancer and its treatment such as fatigue, stress, anxiety or obesity.

Weight gain in adulthood, ${ }^{3}$ overweight, obesity, ${ }^{4}$ as well as lack of physical activity $(\mathrm{PA})^{5}$ and alcohol consumption are risk 
factors commonly associated with risk of breast cancer (mainly in postmenopause for overweight and obesity). ${ }^{6}$ After a diagnosis of breast cancer, obesity appears to double the risk of recurrence and death in these patients, regardless of the state of menopause. ${ }^{78}$ Weight gain after diagnosis is also recognised as a risk factor for increased risk of recurrence and mortality and increases the risk of comorbidities. ${ }^{89}$ In contrast, PA performed appropriately could improve many prognostic factors as well as survival in women after breast cancer. Several large cohort studies of women with breast cancer have shown an average reduction of $45 \%$ mortality associated with moderate exercise compared to a sedentary lifestyle. ${ }^{10-14}$ Randomised control trials have also shown that PA improves fitness, quality of life, self-esteem and treatment adherence and reduces fatigue. ${ }^{7} 1516$ Thus, maintaining an optimal weight through a balanced diet combined with regular PA appears to be effective protective behaviour against breast cancer as well as comorbidity or mortality after a diagnosis of breast cancer. ${ }^{11}$

Observational cohorts of patients with breast cancer conducted in France have shown that currently almost half of the French patients are overweight or obese at diagnosis, ${ }^{9} 17$ and that nearly half of them gain weight within 1 year after chemotherapy. ${ }^{17}$ Patients treated for breast cancer practice less physical exercise of moderate to vigorous intensity than the healthy population for a similar energy expenditure. ${ }^{18}$ Indeed, reduced PA is a factor that is most likely implicated in weight gain among women receiving adjuvant chemotherapy for localised breast cancer. ${ }^{19-21}$ Given the poor prognosis associated with overweight, weight gain and insufficient $\mathrm{PA}$, supportive care including $\mathrm{PA}$ and dietetic care is necessary in patients with breast cancer.

Despite the encouraging results of mainly North American studies on the impact of PA after breast cancer, no intervention studies of PA were conducted until recently in France. ${ }^{22}$ Therefore, our aim was to transpose the international evidence to the French population and context, given the major cultural differences concerning food, the practice of PA and other lifestyle-related factors between the French and the North American populations. In this article, we have described the design of the randomised controlled trial Programme pour une Alimentation Saine et une Activité Physique Adaptée pour les patientes atteintes d'un cancer du Sein (PASAPAS, meaning 'Programme for healthy eating and adapted PA for patients with breast cancer' in English). This study aims at evaluating the feasibility in human, material and financial terms of implementing an individualised programme of dietary counselling and adapted PA (APA) in a French population of women being treated for breast cancer. The APA intervention programme was tailored to prevent the risk of overweight, obesity or weight gain and to maintain and/or increase the patients' PA level.

The main objective of the PASAPAS study is to evaluate, in a cohort of adult patients with a first localised (ie, non-metastatic) breast cancer, the feasibility of implementing an APA intervention for 6 months, in addition to a dietary management, concomitant with the prescription of a first line of adjuvant chemotherapy (which lasts between 12 and 18 weeks according to the protocol). The secondary objectives are to: (1) assess the acceptability of the intervention and randomisation and the recruiting capabilities; (2) assess the patients' adherence to the intervention programme and analyse the reasons for non-adherence; (3) verify the adequacy between the procedures of the intervention programme (APA and dietetic), as well as the data collection and management, and the constraints of implementing the study in real conditions; (4) describe the patients' satisfaction with the dietetic and APA programmes; (5) perform a health economics analysis; (6) describe the baseline state and evolution of a number of variables (PA level and profile, dietary intake, anthropometrics, body composition, lipid profiles, quality of life, body satisfaction/self-perception, self-esteem, depression, anxiety); (7) finally, as part of a joint biological study, describe the metabolomic profiles, lipidomic profiles, as well as the expression of endocrine factors and adipokines linked to breast cancer at initial diagnosis (ie, comparing between tumour and circulating blood before surgery in a cross-sectional study) and metabolic changes during the interventions (longitudinal study).

\section{METHODS AND DESIGN \\ Study design}

The PASAPAS study is an interventional, controlled, randomised, single-centre, open-label, parallel-group study with two arms: an intervention (or 'APA') group where patients are invited to a 6-month exercise programme in supervised groups in addition to the usual and personalised dietetic care; a control group receiving the usual dietetic care and PA national guidelines. The 6-month intervention is followed by a 6-month monitoring, that is a 12-month study duration for each participant. The enrolment is planned over a period of 24 months, which corresponds to a total study duration of 36 months.

The trial was registered on the website http://www. clinicaltrials.gov (registration number: NCT01331772).

\section{STUDY PARTICIPANTS}

\section{Inclusion criteria}

The study sample is composed of adult women, aged 18 years or older and under 75 years, being diagnosed with a first invasive non-metastatic breast carcinoma that has been histologically confirmed, and requiring the prescription for a first line of adjuvant chemotherapy. Other inclusion criteria are: being treated in the clinical site (Léon Bérard Cancer Centre, Lyon, France); being able to participate in the APA intervention and providing a medical certificate of no contraindications to exercise issued by the general practitioner, the referring physician or the main investigator physician; living 
within a $60 \mathrm{~km}$ perimeter and agreeing to support travel expenses to attend group exercise sessions; available and willing to participate in the PASAPAS study for the duration of the programme (6 months) and during the postprogramme monitoring ( 6 months); able to understand, read and write French; affiliated with a social security system; having dated and signed informed consent.

\section{Non-inclusion criteria}

Non-inclusion criteria are woman with metastatic or inflammatory breast cancer; personal history or coexistence of another primary carcinoma (except another in situ carcinoma regardless of the site and/or basal cell skin carcinoma and/or non-mammary cancer in complete remission for more than 5 years); contraindications to exercise according to the investigator such as serious or unstable cardiac or respiratory pathology, uncontrolled diabetes, bone metastases and severe osteoporosis; state of severe malnutrition under the criteria of the French National Health Authority ${ }^{23}$; personal history of eating disorders; unable to be followed for medical, social, family, geographical or psychological reasons, for the study duration; deprived of their liberty by court or administrative decision; pregnant or nursing or of childbearing age without effective contraception during the study.

\section{RECRUITIMENT AND RANDOMISATION}

Patients eligible for the study are identified during the multidisciplinary meeting following surgery. They are presented and offered the study protocol by the oncologist during the consultation performed for planning the chemotherapy (prechemotherapy consultation). If a

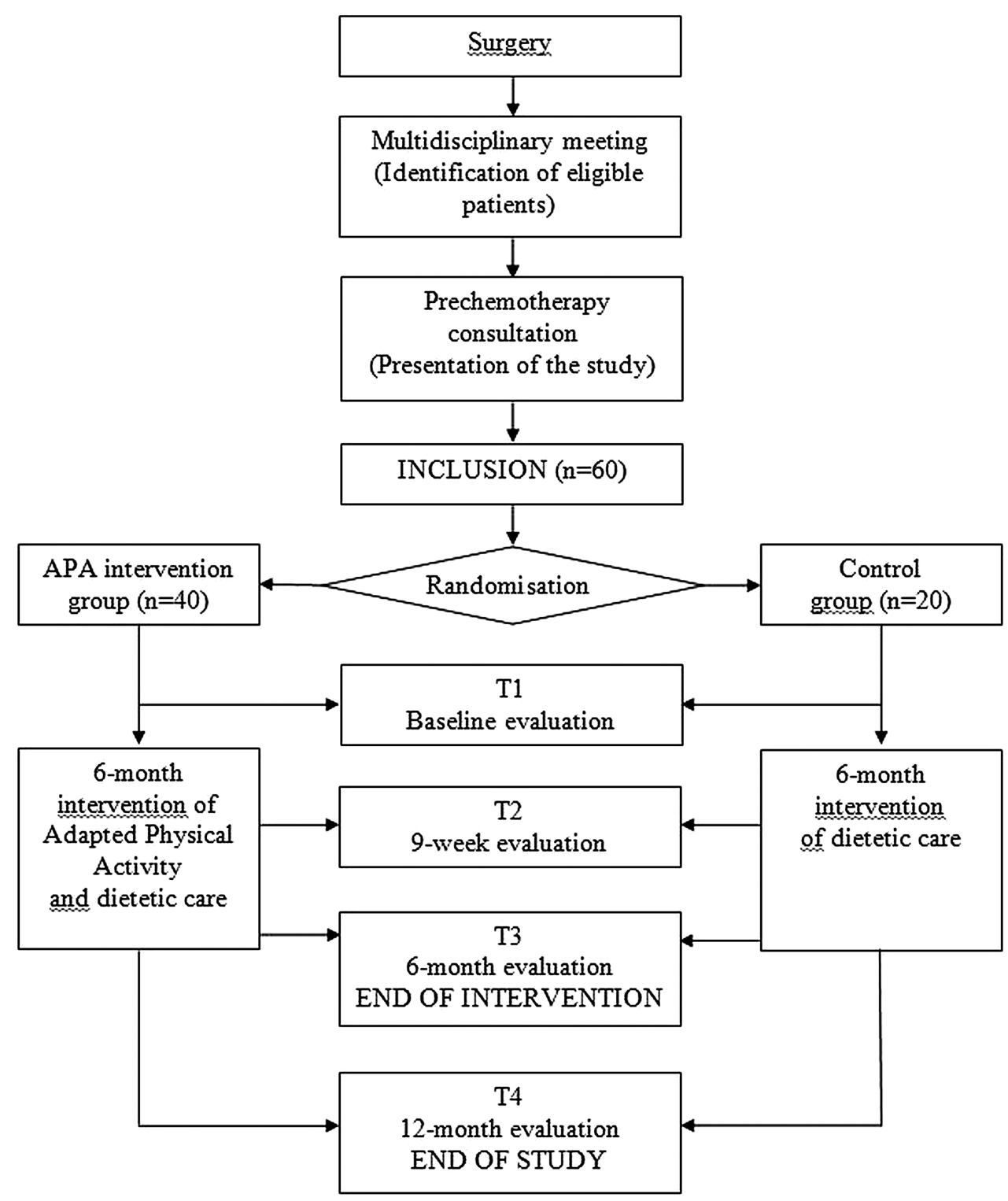

Figure 1 Flow chart of participants through the Programme pour une Alimentation Saine et une Activité Physique Adaptée pour les patientes atteintes d'un cancer du Sein (PASAPAS) study. 
patient agrees to participate, she has to sign an informed consent form. Inclusion and randomisation are conducted within 15 days before the start of chemotherapy (figure 1). Participants are randomly allocated between the APA intervention arm and the control arm following a 2:1 ratio in favour of APA intervention.

\section{INTERVENTIONS}

Dietetic intervention

All participants (intervention as well as control arms) benefit from dietetic and PA support, according to the recommendations of the second French National Health and Nutrition Program (Plan National Nutrition Santé 2, PNNS2). ${ }^{24}$ At the first visit (beginning of adjuvant chemotherapy), the participants meet the dietician and receive individualised dietary counselling according to dietary analysis, as well as print material (entitled "Tips for a healthy diet and practicing physical activity" and labelled in accordance with the PNNS2). Moreover, dietary management is offered to each participant; in case of acceptance, dietetic consultations are offered on the patient's request for the duration of the intervention (6 months).

\section{APA intervention}

Women randomised to the intervention arm are offered, in addition to dietary management (see Dietetic intervention section), an opportunity to participate in group sessions of APA over a period of 6 months, including: (1) 2 sessions/week throughout the duration of chemotherapy (four to six 3-week courses depending on the protocol, ie, for 12-18 weeks), except during the first week of each course as a recovery from exercise and to take into account fatigue and other side effects related to chemotherapy; (2) 3 sessions/week after cessation of chemotherapy, until the end of the 6-month intervention (ie, for 8-14 weeks). For the duration of chemotherapy, the APA programme includes one session of Nordic walking outdoors and one session of aerobic fitness indoors (combining cardiovascular workouts such as step, muscle strengthening and stretching or relaxation) per week. After chemotherapy, the APA programme includes two sessions of Nordic walking and one session of aerobic fitness per week.

The group sessions of a maximum of eight patients are supervised by an APA professional. Exercises consist of aerobics of moderate intensity (4-6 Metabolic Equivalents of Task, METs) adapted according to the initial PA profile of each participant. The APA programme is an individualised exercise according to comorbidities and defined by the PA level (jointly defined by the PA profile or time spent in PAs of different intensities and by aerobic capacity) and the body composition status. Each session comprises a warm-up of $10 \mathrm{~min}$, a core period (20-30 min for aerobic fitness exercise or 30-40 min for Nordic walking) and a cooldown period of $10 \mathrm{~min}$. The progressive increase in workload is conducted by an increased frequency (from two sessions early in the programme to three sessions at the end of the programme), then the actual duration and lastly (after the end of chemotherapy) the intensity depending on the patient's desire and capacity. In all cases, the intensity of activity remains less than $80 \%$ of the age-adjusted maximal heart rate (equal to 220-age expressed in years).

Attending the group sessions is required to determine the compliance of each participant with the APA programme, but participants are free to practice more exercise individually or attend additional group sessions. In case a participant cannot attend a group session (for medical or personal reasons or due to exceptional circumstances such as holidays), she must replace it during the same week with an individual session of another aerobic exercise (eg, Nordic walking, free swimming, hiking, biking), which is planned in advance with the APA professional; this intends to avoid a decrease in physical fitness. To verify attendance in the programme, exercise is recorded in a notebook and the number of steps is recorded by a pedometer given to participants.

If side effects occur during 3-week cycles of chemotherapy, patients may be prescribed a weekly chemotherapy (mainly paclitaxel). The APA programme remains the same for these patients, meaning they have no session during each weekly chemotherapy cycle and resume APA at the end of chemotherapy, but they can attend group sessions if they wish.

\section{DATA COLLECTION AND EVALUATIONS}

As part of this study, patients of both arms are followed over a total duration of 12 months (ie, for the 6-month dietetic and APA intervention programme and a 6-month follow-up after the intervention). Four evaluations are performed (see figure 1): at baseline (T1, day 1 of chemotherapy), at 9 weeks (T2, day 1 of the fourth 3 -week course of chemotherapy), at 26 weeks \pm 1 week (T3, end of the intervention 6 months after initiation of chemotherapy) and at 52 weeks \pm 4 weeks (T4, end of the 6-month follow-up postintervention), which corresponds to the end of the study for patients. All the following data are collected at each evaluation, except dietary and psychological assessments that are performed at T1, T3 and T4 only and patients' satisfaction for the intervention in both arms that is evaluated at T3 only.

\section{Physical activity}

The level and profile of PA are assessed at each evaluation using the Physical Activity Questionnaire Activité Physique (PAQAP) questionnaire ${ }^{25}$ administered by interview; the International Physical Activity Questionnaire (IPAQ) is filled at the same time for international comparison. PA before the time of diagnosis is also assessed retrospectively at $\mathrm{T} 1$, to distinguish between activities that are no longer practiced since diagnosis and new activities practiced since surgery. The 
PAQAP questionnaire provides the estimated aerobic capacity $\left(V \mathrm{O}_{2 \max }, \mathrm{mL} / \mathrm{min} / \mathrm{kg}\right)$, usual average daily energy expenditure $(\mathrm{kJ} /$ day), PA profile (ie, time spent in activities of the following intensities: below 2 METs or sedentary, 2-2.9 METs, 3-3.9 METs, 4-5.9 METs, 6-9.9 METs and $>10$ METs) and time spent sitting and lying.

\section{Anthropometrics and body composition}

The following anthropometric data are measured at each evaluation: weight $(\mathrm{kg})$ using the same scale throughout the study, height $(\mathrm{cm})$ using a fathom, waist circumference $(\mathrm{cm})$ and hip circumference $(\mathrm{cm})$ using a measuring tape. For standardising measurements, waist circumference is measured midway between the last floating rib and the iliac crest and the hip circumference is measured at the tip of the pubis. Body mass index (BMI) is calculated as weight $(\mathrm{kg})$ divided by the square of height $(\mathrm{m})$. Weight 1 month ago (close to surgery), 6 months ago (close to diagnosis) and 1 year ago is also declared retrospectively at baseline.

Risk of metabolic diseases is identified for this population as follows: metabolic risk ${ }^{26}$ if waist circumference to height ratio $>0.5$; insulin-resistance risk ${ }^{27}$ if waist circumference $>80 \mathrm{~cm}$; and additionally cardiovascular risk ${ }^{28}$ if waist circumference $>88 \mathrm{~cm}$.

Body composition is evaluated using a multifrequency bioelectrical impedance analysis (QuadScan4000, Bodystat; measure duration of about $30 \mathrm{~s}$, after lying for $10 \mathrm{~min})$ that provides body fat $(\%, \mathrm{~kg})$, lean mass $(\%, \mathrm{~kg})$, dry lean mass, basal metabolic rate, basal metabolic rate to weight ratio and body water $(\%, 1$; total, intracellular, extracellular). Normal-weight obese women are identified using the waist circumference and more particularly the percentage of body fat in comparison to BMI status. ${ }^{29} 30$ Body composition is also assessed at the time of diagnosis by analysing a CT scan (systematically performed for clinical management) at the L3 lumbar position and measuring the area occupied by adipose (subcutaneous and visceral) and lean tissue (Slice-O-Matic Software, V.4.3, Tomovision).

\section{Nutritional intake}

Dietary intake is assessed by a 3-day food diary (2-weekdays and 1-weekend day) completed by the patient in the week preceding the evaluation and reviewed by the dietician using standardised portions. ${ }^{31}$ A dietary analysis using the GENI software (Micro 6, Villers-les-Nancy, France, V.7.4) provides the caloric, macronutrient and fluid intake. The dietician also assesses the usual consumption of alcoholic beverages over the preceding 6 months, the use of dietary supplements and the compliance with the PNNS2 guidelines. ${ }^{32}$

\section{Biological samples}

A fasting blood sample is collected at each evaluation as part of the biological study (in particular, blood will be drawn before infusion of chemotherapy at T1 and T2). Two tubes are drawn each time (one dry tube of $10 \mathrm{~mL}$ and one EDTA tube of $10 \mathrm{~mL}$ ). Blood parts (ie, serum, plasma and buffy coat) are separated and stored in aliquots of $300 \mu \mathrm{L}$ in liquid nitrogen. After completion of the study, the following analyses will be performed longitudinally:

- Levels of plasma biomarkers of dietary intake of antioxidants (total carotenoids, lycopene, $\beta$-carotene, $\alpha$-tocopherol);

- Lipidomics analysis: complete profile of about 50 fatty acids, including the circulating levels of saturated, monounsaturated cis, n- 6 polyunsaturated, n-3 polyunsaturated and trans fatty acids, as well as the $\omega 3$ to $\omega 6$ ratio;

- Levels of cytokinic factors (leptin, adiponectin, tumour necrosis factor- $\alpha$, transforming growth factor- $\beta$ ) and endocrine factors (insulin, insulin-like growth factor 1, sex hormone-binding globulin) in plasma;

- Metabolomics analysis: measurement of serum metabolites identified by the profile obtained by nuclear MR such as amino acids, sugars, organic acids and all identifiable metabolites of molecular weight below $1 \mathrm{kDa}$; identification of multivariate metabolic profile characteristics for each study arm.

For the cross-sectional study at surgery, a tumour sample (frozen if tumour size $\geq 20 \mathrm{~mm}$ ) and a circulating blood sample that were collected whenever possible during surgery will be obtained retrospectively from the clinic biobank. Then, a complete profile of fatty acids, measurement of cytokinic and endocrine factors and metabolomics analysis will also be performed on them.

Finally, levels of fasting glucose, albumin, lipids (total cholesterol and its high-density lipoprotein and lowdensity lipoprotein fractions, triglycerides) are measured at each evaluation.

\section{Psychological criteria}

Psychological criteria are assessed at each evaluation using self-administered questionnaires: quality of life using the European Organisation for Research and Treatment of Cancer Quality of Life Questionnaire Core 30 (EORTC QLQ-C30) questionnaire with its module BR-23 specific for breast cancer ${ }^{33} 34$ and the Medical Outcomes Study 36-Item Short-Form Health Survey (MOS SF-36) questionnaire ${ }^{35} 36$ (V.4.0 in the French language); body satisfaction and global self-perception using the Questionnaire de Satisfaction Corporelle et de Perception Globale de Soi (QSCPGS) questionnaire ${ }^{37}$; self-esteem using the Scale of Rosenweig ${ }^{38}{ }^{39}$; depression using the Beck Depression Inventory (BDI) questionnaire ${ }^{40-42}$; and anxiety using the State Trait Anxiety Inventory (STAI) questionnaire. ${ }^{43} 44$

\section{Patients' satisfaction}

Overall satisfaction of the patients for the intervention in both arms is assessed at the end of the programme (T3) using a graduated scale. 


\section{Other data collection}

Clinical data, demographic data, medical history and reproductive history are collected at baseline. Tobacco use is collected at each evaluation.

In the intervention group, adherence to the APA sessions (primary outcome) is monitored by the APA professionals and reasons for non-adherence are recorded.

All adverse events (except haematological adverse events, gastrointestinal and hepatobiliary events that are frequently linked to adjuvant treatment of cancer) and all concomitant medications are reported by the patients continuously during the 6-month intervention period using a booklet.

Globally, the duration of each evaluation is estimated to be approximately $2 \mathrm{~h}$ /patient (10 min for anthropometric measurements, 10 min for the rest necessary for impedance measurements, 45-60 min for PA assessment and 35-45 min for the dietary evaluation).

\section{STUDY OUTCOMES}

The primary endpoint will be the proportion of patients in the intervention group who participate in at least two group sessions of APA per week for the duration of the APA programme (ie, 26 weeks from day 1 of chemotherapy), except during the first week of each course (see APA intervention section).

Secondary endpoints are

1. Assessment of the acceptability of the intervention and randomisation (proportion of eligible and invited patients who volunteer to participate in the study, rate of recruitment), as well as the qualitative analysis of barriers to recruitment (eg, competition with other therapeutic trials, geographic distance, etc).

2. Assessment of patients' compliance (percentage of patients who complete the full APA programme), as well as the qualitative analysis of the reasons for non-compliance.

3. Formalisation of the APA and dietetic intervention programme and the data collection and management process to implement in a future study (adequacy between procedures and study implementation in real conditions).

4. Score of overall satisfaction of the patients with the intervention, at the end of the intervention.

5. Costs supported/avoided for healthcare providers.

6. Baseline and changes in nutritional intake, PA level and profile, anthropometrics, body composition and psychological parameters.

7. Baseline and changes in biological parameters (metabolome, lipidome, cytokines, endocrine factors) along the intervention, as well as their comparison between the tumour and circulating blood at the time of initial diagnosis (before surgery).

\section{WITHDRAWAL FROM THE PASAPAS STUDY}

Participants may interrupt the study prematurely for the following various reasons: a relapse of disease; a decision of the promoter or the investigator; death of the patient. Except in the last case, a final evaluation will be performed.

\section{HEALTH ECONOMICS ANALYSIS}

The literature shows a growing interest in health economics questions relating to PA for patients with cancer. ${ }^{45} 46$ The economics impact of an APA intervention in patients with localised invasive breast cancer undergoing chemotherapy will be assessed based on a cost analysis and a budget impact analysis.

\section{Cost analysis}

Costs will be assessed prospectively for each patient. The identification, measurement and valuation of costs will be performed based on the hospital's point of view. Hence, cost computations will focus on inpatient, outpatient and home care. Length of stay within a conventional medical unit, rehabilitation unit, home care, etc will be identified and multiplied by their respective unit costs. The time frame will be 12 months. Mean costs will be assessed for APA intervention as well as control groups. All costs will be related to 2013 and will be given in euros.

\section{Budget impact analysis}

The hospital perspective will be retained and a 1-year baseline period presented. Target and prevalent populations will be estimated based on this study and the Léon Bérard Cancer Centre data.

\section{STATISTICAL ANALYSIS}

\section{Sample size}

The number of patients to include was defined empirically. A sample size of 60 patients seems sufficient to explore the feasibility of such a programme and to identify the potential corrective measures to take into account in interventions in the context of a future randomised clinical trial for efficacy. To emphasise the size of the group of patients supported by the APA intervention (main target of the study), an unbalanced randomisation (2:1 ratio) has been proposed.

\section{Statistical analyses}

The analysis will be performed in the intent-to-treat population, including all patients randomised in the arm allocated by randomisation, in order to limit the possibility of bias associated with participants not receiving the intervention they were allocated for. Baseline characteristics will be summarised by treatment arms using standard descriptive statistics. Primary outcome will be described as the proportion of patients who participate in at least two planned APA sessions per week, with its $95 \%$ CI. Participation in the APA programme will be evaluated by the rate of patients who participate in all APA sessions and reasons for non-participation will be described. Tolerance analysis will be performed by studying the rate and its $95 \% \mathrm{CI}$ of patients who had at least one adverse event in the APA arm. 
For secondary outcome variables, changes in the anthropometric measurements, body composition variables, PA profile and dietary, psychological and biological variables will be analysed by the absolute and relative variations of each of these endpoints between randomisation (initial assessment at T1) and follow-up assessments (T2-T4). These variations will be compared between both arms of randomisation using a nonparametric test. A method for imputing missing data will be considered if necessary.

\section{Health economics analysis}

Descriptive statistics will be used to present costs. Ninety-five per cent CIs will be calculated. Costs will be compared using the Mann-Whitney test. When necessary, multiple regression analyses will be performed to examine the relationship between costs and a range of potentially explanatory clinical variables.

\section{ETHICS CONSIDERATIONS AND DISSEMINATION}

The PASAPAS study was approved by the French ethics Committee (Comité de Protection des Personnes Sud-Est IV), the National Security Agency of Medicines and Health Products that applies for biomedical studies (Agence Nationale de Sécurité du Médicament et des produits de santé) and the French National Committee on Informatics and Privacy (Commission Nationale de l'Informatique et des Libertés). All participants give written informed consent. The study imposes no risks on the participants and the APA programme is safe.

The study findings will be widely disseminated through the scientific public by publication in international peer-reviewed journals and presentation in national and international conferences. They will also be useful for optimising the intervention programme and serve as a foundation for a future full-scale randomised controlled trial.

\section{DISCUSSION}

The goal of this article was to describe the protocol of a multidisciplinary 6-month intervention study of APA and dietary management for patients with breast cancer undergoing adjuvant chemotherapy. This single centre feasibility study is designed to evaluate the feasibility of implementing an APA programme and test the study process prior to a multicentre full-scale randomised controlled trial testing the effectiveness of an APA programme during breast cancer treatment on survival-related outcomes. Inclusions in the PASAPAS study started in June 2011 and the study will be closed in June 2014.

The optimal management for an improved PA level of a healthy individual appears to be at least three sessions/week, 30-60 min each and of moderate intensity, allowing a sufficient dose of exercise. The intensity and type of exercise included in the APA programme for patients with breast cancer were based on literature evidence that recommends moderate-intensity exercise that combines aerobic and resistance exercise. ${ }^{47} 48$ Therefore, sessions of aerobic and resistance exercise of at least 4 MET intensity, such as Nordic walking and aerobic fitness, were planned two to three times a week to permit a progressive increase in exercise frequency. An interval of at least $48 \mathrm{~h}$ between sessions allows a complete recovery and the sessions are close enough to promote metabolic responses. ${ }^{49}$ The APA programme is individualised in terms of intensity (above 4 METs) and duration of the sessions according to the initial PA level, anthropometrics, body composition and comorbidities. APA sessions are supervised by trained APA professionals (similar to exercise physiologists).

The APA programme was designed to be feasible for patients with breast cancer undergoing treatment and to obtain a metabolic response to exercise. The APA programme aims: (1) to prevent physical deconditioning (ie, to preserve the initial PA level or improve it if it is insufficient or below recommendations); (2) to avoid weight gain, which is common during or after breast cancer treatment; (3) to improve the quality of life; (4) and to reduce fatigue. Improvements are expected in terms of body composition (maintenance or increase in lean mass, no increase or decrease in body fat), cardiorespiratory capacity, balance and flexibility. PA has been shown to be safe in patients with cancer. ${ }^{50}$

The strengths of the study include, first, the PA intervention that is personalised and supervised by trained professionals. Group sessions provide support to patients with cancer for being physically active and following an exercise programme. Moreover, meeting other patients with cancer with similar health conditions and creating social links or friendship are reinforcement techniques for successfully modifying lifestyle habits such as PA or sedentary behaviours. Second, the timing of the APA intervention during adjuvant treatment makes the study original. Most PA interventions in patients with cancer are performed after treatment ${ }^{51-53}$ and are not necessarily randomised. ${ }^{54}$ However, growing evidence shows the necessity to maintain or start PA as soon as possible after a cancer diagnosis and avoid loss of physical fitness. ${ }^{55} 56$ Third, the 6-month programme is relatively long compared to other programmes published in the literature and may help patients sustain in a regular practice of PA. Fourth, a biological substudy will provide insight into the mechanisms involved in cancer and during an exercise intervention. In spite of the small size of this feasibility study, biological results will raise mechanistic hypotheses for a future large-scale randomised controlled trial. Fifth, a medicoeconomic evaluation is original and will guide the institution in integrating PA in the care of patients with cancer. Finally, the 6-month programme is relatively long compared to other programmes published in the literature and may help patients sustain in a regular practice of PA.

The authors also recognise the limitations of the study. The small sample size does not provide statistical power to test the efficacy of the APA intervention in 
modifying prognosis factors, but it is sufficient for the primary goal of the study to test the feasibility of implementing the programme. Feasibility studies are often of very small size indeed. ${ }^{57}$ Because the APA programme is supervised and performed locally, the target population is limited to patients living in the close area of the clinic. Recruitment in the study is strongly limited for this reason.

Given the large and growing population affected by breast cancer worldwide and the important benefit of PA on breast cancer survival identified in observational studies, it is now necessary to study the impact of PA on disease-free survival and overall survival in a multicenter randomised study design. However, studying overall survival might be difficult, if not impossible, to achieve given the relatively good prognosis of breast cancer ${ }^{59}$ and the growing efforts of national nutrition and PA prevention programmes. Therefore, a valid intermediate survival endpoint might be necessary, which might be related to body composition or a composite endpoint based on biological mechanisms. ${ }^{60}$

\section{Author affiliations \\ ${ }^{1}$ Department of Cancer and Environment, Léon Bérard Cancer Centre, Lyon, France \\ ${ }^{2}$ Centre for Research and Innovation on Sport EA647, University Claude Bernard Lyon 1, Villeurbanne, France \\ ${ }^{3}$ EA4129 'Santé, Individu, Société', University Lyon 1, Lyon, France \\ ${ }^{4}$ Unit of Biostatistics and Therapeutic Evaluation, Léon Bérard Cancer Centre, \\ Lyon, France \\ 5University Lyon 2, CNRS, GATE Lyon Saint-Etienne, Ecully, France \\ ${ }^{6}$ Department of Medical Oncology, Léon Bérard Cancer Centre, Lyon, France \\ ${ }^{7}$ Nutrition Unit-DISSPO, Léon Bérard Cancer Centre, Lyon, France}

Acknowledgements The authors are grateful to $\mathrm{Dr}$ Véronique Chajès, $\mathrm{Pr}$ Marie-Paule Vasson, Dr Ruth Rimokh, Pr Joëlle Goudable and Dr Bénédicte Elena-Herrmann for their contribution during the development of the biological study.

Contributors PB conceived the idea for the study. All authors contributed to the design of the study. A-MF and SEB contributed to the development of the adapted physical activity programme and are responsible for the physical activity analysis. MT, A-MF, A-SK-L, SG and VB-B contributed to the set-up of the study. SC will be responsible for the statistical analysis. LP will be responsible for the medicoeconomic analysis. MT wrote the manuscript. All authors read, revised and approved the final manuscript.

Funding The PASAPAS trial is financially supported by the French Cancer Institute ('Institut National du Cancer', grant no. 2010-228/VO-HO), the 'Ligue contre le cancer', the 'Fondation de France' (grant no. 2010-15024) and the 'Cancéropôle Lyon Auvergne Rhône-Alpes'. A-MF was supported by the French Ministry of Research during her doctoral training.

\section{Competing interests None.}

Ethics approval Ethics approval was provided by the Comité de Protection des Personnes Sud-Est IV.

Provenance and peer review Not commissioned; peer reviewed for ethical and funding approval prior to submission.

Open Access This is an Open Access article distributed in accordance with the Creative Commons Attribution Non Commercial (CC BY-NC 3.0) license, which permits others to distribute, remix, adapt, build upon this work noncommercially, and license their derivative works on different terms, provided the original work is properly cited and the use is non-commercial. See: http:// creativecommons.org/licenses/by-nc/3.0/

\section{REFERENCES}

1. Jemal A, Bray F, Center MM, et al. Global cancer statistics. $C A$ Cancer J Clin 2011;61:69-90.

2. INCa. La situation du cancer en France en 2011. 2011.

3. Lahmann PH, Schulz M, Hoffmann K, et al. Long-term weight change and breast cancer risk: the European prospective investigation into cancer and nutrition (EPIC). Br J Cancer 2005;93:582-9.

4. Calle EE, Rodriguez C, Walker-Thurmond K, et al. Overweight, obesity, and mortality from cancer in a prospectively studied cohort of U.S. adults. N Engl J Med 2003;348:1625-38.

5. Friedenreich $\mathrm{CM}$, Cust AE. Physical activity and breast cancer risk: impact of timing, type and dose of activity and population subgroup effects. Br J Sports Med 2008;42:636-47.

6. WCRF/AICR. Food, nutrition, physical activity, and the prevention of cancer: a global perspective. Washington, DC: AICR, 2007.

7. Speck RM, Courneya KS, Masse LC, et al. An update of controlled physical activity trials in cancer survivors: a systematic review and meta-analysis. J Cancer Surviv 2010;4:87-100.

8. Ibrahim EM, Al-Homaidh A. Physical activity and survival after breast cancer diagnosis: meta-analysis of published studies. Med Oncol 2011;28:753-65.

9. Majed B, Moreau T, Senouci K, et al. Is obesity an independent prognosis factor in woman breast cancer? Breast Cancer Res Treat 2008;111:329-42.

10. Holmes MD, Chen WY, Feskanich D, et al. Physical activity and survival after breast cancer diagnosis. JAMA 2005;293:2479-86.

11. Pierce JP, Stefanick ML, Flatt SW, et al. Greater survival after breast cancer in physically active women with high vegetable-fruit intake regardless of obesity. J Clin Oncol 2007;25:2345-51.

12. Holick CN, Newcomb PA, Trentham-Dietz A, et al. Physical activity and survival after diagnosis of invasive breast cancer. Cancer Epidemiol Biomarkers Prev 2008;17:379-86.

13. Irwin ML, Smith AW, McTiernan A, et al. Influence of pre- and postdiagnosis physical activity on mortality in breast cancer survivors: the health, eating, activity, and lifestyle study. J Clin Oncol 2008;26:3958-64

14. Friedenreich CM, Gregory J, Kopciuk KA, et al. Prospective cohort study of lifetime physical activity and breast cancer survival. Int $J$ Cancer 2009;124:1954-62.

15. Cramp F, Byron-Daniel J. Exercise for the management of cancer-related fatigue in adults. Cochrane Database Syst Rev 2012; (11):CD006145.

16. Duijts SF, Faber MM, Oldenburg HS, et al. Effectiveness of behavioral techniques and physical exercise on psychosocial functioning and health-related quality of life in breast cancer patients and survivors-a meta-analysis. Psychooncology 2011;20:115-26.

17. Trédan $\mathrm{O}$, Bajard $\mathrm{A}$, Meunier $\mathrm{A}$, et al. Body weight change in women receiving adjuvant chemotherapy for breast cancer: a French prospective study. Clin Nutr 2010;29:187-91.

18. Guichard JB, Garet M, Auberdiac P, et al. [Assessment of daily physical activity of breast cancer patients and comparison with two control populations]. Bull Cancer 2011;98:733-40.

19. Demark-Wahnefried W, Hars V, Conaway MR, et al. Reduced rates of metabolism and decreased physical activity in breast cancer patients receiving adjuvant chemotherapy. Am J Clin Nutr 1997;65:1495-501.

20. Irwin ML, Crumley D, McTiernan A, et al. Physical activity levels before and after a diagnosis of breast carcinoma: the Health, Eating, Activity, and Lifestyle (HEAL) study. Cancer 2003;97:1746-57.

21. Harvie MN, Campbell IT, Baildam A, et al. Energy balance in early breast cancer patients receiving adjuvant chemotherapy. Breast Cancer Res Treat 2004;83:201-10.

22. Romieu I, Touillaud M, Ferrari $\mathrm{P}$, et al. Activité physique et survie après cancer [Physical activity and cancer survival]. Bull Cancer 2012;99:979-94.

23. HAS. Evaluation diagnostique de la dénutrition protéino-énergétique des adultes hospitalisés. ANAES, Service des recommandations professionnelles 2003.

24. Ministère de la Santé et des Solidarités. Deuxième Programme national nutrition santé, 2006-2010-Actions et mesures. 1-51. 2006. Paris. 2006.

25. Berthouze-Aranda SE, Aranda P. Un logiciel pour la mesure de l'activité physique : le PAQAP. Sports Sci 2001;16:171-2.

26. Ashwell M, Gibson S. Waist to height ratio is a simple and effective obesity screening tool for cardiovascular risk factors: analysis of data from the British National Diet And Nutrition Survey of adults aged 19-64 years. Obes Facts 2009;2:97-103.

27. Alberti KG, Zimmet P, Shaw J. Metabolic syndrome-a new world-wide definition. A Consensus Statement from the International Diabetes Federation. Diabet Med 2006;23:469-80. 
28. NCEP Expert Panel. Executive summary of the third report of the National Cholesterol Education Program (NCEP) Expert Panel on detection, evaluation, and treatment of high blood cholesterol in adults (Adult Treatment Panel III). JAMA 2001;285:2486-97.

29. DeLorenzo A, Martinoli R, Vaia F, et al. Normal weight obese (NWO) women: an evaluation of a candidate new syndrome. Nutr Metab Cardiovasc Dis 2006;16:513-23.

30. Marques-Vidal P, Chiolero A, Paccaud F. Large differences in the prevalence of normal weight obesity using various cut-offs for excess body fat. e-SPEN 2008;3:e159-62.

31. Su.Vi.Max. Portions alimentaires. Paris: Manuel-photos pour l'estimation des quantités, 2002.

32. Estaquio C, Kesse-Guyot E, Deschamps V, et al. Adherence to the French Programme National Nutrition Sante Guideline Score is associated with better nutrient intake and nutritional status. J Am Diet Assoc 2009;109:1031-41.

33. Aaronson NK, Ahmedzai S, Bergman B, et al. The European Organization for Research and Treatment of Cancer QLQ-C30: a quality-of-life instrument for use in international clinical trials in oncology. J Natl Cancer Inst 1993;85:365-76.

34. Fayers P, Bottomley A. Quality of life research within the EORTC-the EORTC QLQ-C30. European Organisation for Research and Treatment of Cancer. Eur J Cancer 2002;38(Suppl 4):S125-33.

35. Leplege A, Ecosse E, Verdier A, et al. The French SF-36 Health Survey: translation, cultural adaptation and preliminary psychometric evaluation. J Clin Epidemiol 1998;51:1013-23.

36. Wade JE, Sherbourne CD. The MOS 36-item short-form health survey (SF-36). Med Care 1994;30:473-83.

37. Evers L, Verbanck P. [Creation of a body satisfaction and global self-perception questionnaire: the QSCPGS. Norms and first validation]. Encephale 2010;36:21-7.

38. Rosenberg M. Society and the adolescent self-image. Princeton, NJ: Princeton University Press, 1965.

39. Vallières EF, Vallérand RJ. Traduction et validation canadienne-française de l'échelle de l'estime de soi de Rosenberg. Int J Psychol 1990;25:305-16.

40. Beck AT, Ward $\mathrm{CH}$, Mendelson $\mathrm{M}$, et al. An inventory for measuring depression. Arch Gen Psychiatry 1961;4:561-71.

41. Beck AT. Depression inventory. Philadelphia: Center for Cognitive Therapy, 1978.

42. Bourque $P$, Beaudette $D$. Étude psychométrique du questionnaire de dépression de Beck auprès d'un échantillon d'étudiants universitaires francophones. Rev Can Sci comportement 1982;14:211-18.

43. Bruchon-Schweitzer ML, Paulhan I. Manuel pour l'inventaire d'anxiété trait-état (forme Y). Paris: Edition du centre de psychologie appliquée, 1993.

44. Spielberger CD. Manual for the state-trait anxiety inventory (form $Y$ ). Palo Alto, CA: Consulting Psychologist Press, 1983.

45. van Waart $\mathrm{H}$, Stuiver MM, van Harten $\mathrm{WH}$, et al. Design of the Physical exercise during Adjuvant Chemotherapy Effectiveness Study (PACES): a randomized controlled trial to evaluate effectiveness and cost-effectiveness of physical exercise in improving physical fitness and reducing fatigue. BMC Cancer 2010;10:673.
46. Kampshoff CS, Buffart LM, Schep G, et al. Design of the Resistance and Endurance exercise After ChemoTherapy (REACT) study: a randomized controlled trial to evaluate the effectiveness and cost-effectiveness of exercise interventions after chemotherapy on physical fitness and fatigue. BMC Cancer 2010;10:658.

47. Courneya KS, Mackey JR, McKenzie DC. Exercise for breast cancer survivors: research evidence and clinical guidelines. Phys Sportsmed 2002;30:33-42.

48. Courneya KS, Segal RJ, Mackey JR, et al. Effects of aerobic and resistance exercise in breast cancer patients receiving adjuvant chemotherapy: a multicenter randomized controlled trial. J Clin Oncol 2007; $25: 4396-404$

49. Wilmore JH, Costill DL. Physiology of sport and exercise. Champaign, IL: Human kinetics, 1999.

50. Schmitz KH, Courneya KS, Matthews C, et al. American College of Sports Medicine roundtable on exercise guidelines for cancer survivors. Med Sci Sports Exerc 2010;42:1409-26.

51. Irwin ML, Cadmus L, Alvarez-Reeves M, et al. Recruiting and retaining breast cancer survivors into a randomized controlled exercise trial: the Yale Exercise and Survivorship Study. Cancer 2008;112(11 Suppl):2593-606.

52. Kwiatkowski F, Mouret-Reynier MA, Duclos M, et al. Long term improved quality of life by a 2-week group physical and educational intervention shortly after breast cancer chemotherapy completion. Results of the 'Programme of Accompanying women after breast Cancer treatment completion in Thermal resorts' (PACThe) randomised clinical trial of 251 patients. Eur J Cancer 2013;49:1530-8.

53. Saarto $\mathrm{T}$, Penttinen $\mathrm{HM}$, Sievanen $\mathrm{H}$, et al. Effectiveness of 12-month exercise program on physical performance and quality of life of breast cancer survivors. Anticancer Res 2012;32:3875-84.

54. Schmitz KH, Ahmed RL, Hannan PJ, et al. Safety and efficacy of weight training in recent breast cancer survivors to alter body composition, insulin, and insulin-like growth factor axis proteins. Cancer Epidemiol Biomarkers Prev 2005;14:1672-80.

55. NCCN. Clinical practice guidelines in oncology for survivorship. 14 Mar 2013.

56. Rock CL, Doyle C, Demark-Wahnefried W, et al. Nutrition and physical activity guidelines for cancer survivors. CA Cancer J Clin 2012:62:243-74.

57. Campbell KL, Van Patten CL, Neil SE, et al. Feasibility of a lifestyle intervention on body weight and serum biomarkers in breast cancer survivors with overweight and obesity. J Acad Nutr Diet 2012;112:559-67.

58. Portela AL, Santaella CL, Gomez CC, et al. Feasibility of an exercise program for Puerto Rican women who are breast cancer survivors. Rehabil Oncol 2008;26:20-31.

59. Ballard-Barbash $\mathrm{R}$, Hunsberger S, Alciati $\mathrm{MH}$, et al. Physical activity, weight control, and breast cancer risk and survival: clinical trial rationale and design considerations. J Natl Cancer Inst 2009;101:630-43.

60. Ballard-Barbash R, Friedenreich CM, Courneya KS, et al. Physical activity, biomarkers, and disease outcomes in cancer survivors: a systematic review. J Natl Cancer Inst 2012;104:815-40. 